\title{
Front Matter: Volume 9342
}

, "Front Matter: Volume 9342," Proc. SPIE 9342, Solid State Lasers XXIV: Technology and Devices, 934201 (16 April 2015); doi: 10.1117/12.2183961

SPIE. Event: SPIE LASE, 2015, San Francisco, California, United States 


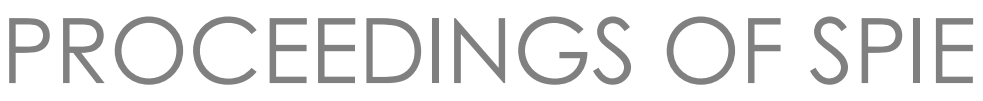

\title{
Solid State Lasers XXIV: Technology and Devices
}

\author{
W. Andrew Clarkson \\ Ramesh K. Shori \\ Editors
}

8-10 February 2015

San Francisco, California, United States

Sponsored and Published by

SPIE 
The papers included in this volume were part of the technical conference cited on the cover and title page. Papers were selected and subject to review by the editors and conference program committee. Some conference presentations may not be available for publication. The papers published in these proceedings reflect the work and thoughts of the authors and are published herein as submitted. The publisher is not responsible for the validity of the information or for any outcomes resulting from reliance thereon.

Please use the following format to cite material from this book:

Author(s), "Title of Paper," in Solid State Lasers XXIV: Technology and Devices, edited by W. Andrew Clarkson, Ramesh K. Shori, Proceedings of SPIE Vol. 9342 (SPIE, Bellingham, WA, 2015) Article CID Number.

ISSN: 0277-786X

ISBN: 9781628414325

\section{Published by}

\section{SPIE}

P.O. Box 10, Bellingham, Washington 98227-0010 USA

Telephone +1 3606763290 (Pacific Time) · Fax +1 3606471445

SPIE.org

Copyright @ 2015, Society of Photo-Optical Instrumentation Engineers.

Copying of material in this book for internal or personal use, or for the internal or personal use of specific clients, beyond the fair use provisions granted by the U.S. Copyright Law is authorized by SPIE subject to payment of copying fees. The Transactional Reporting Service base fee for this volume is $\$ 18.00$ per article (or portion thereof), which should be paid directly to the Copyright Clearance Center (CCC), 222 Rosewood Drive, Danvers, MA 01923. Payment may also be made electronically through CCC Online at copyright.com. Other copying for republication, resale, advertising or promotion, or any form of systematic or multiple reproduction of any material in this book is prohibited except with permission in writing from the publisher. The CCC fee code is 0277-786X/15/\$18.00.

Printed in the United States of America.

Publication of record for individual papers is online in the SPIE Digital Library.

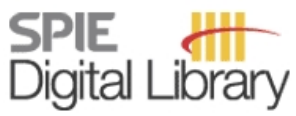

SPIEDigitalLibrary.org

Paper Numbering: Proceedings of SPIE follow an e-First publication model, with papers published first online and then in print. Papers are published as they are submitted and meet publication criteria. A unique citation identifier (CID) number is assigned to each article at the time of the first publication. Utilization of CIDs allows articles to be fully citable as soon as they are published online, and connects the same identifier to all online, print, and electronic versions of the publication. SPIE uses a six-digit CID article numbering system in which:

- The first four digits correspond to the SPIE volume number.

- The last two digits indicate publication order within the volume using a Base 36 numbering

system employing both numerals and letters. These two-number sets start with 00, 01, 02, 03, 04, $05,06,07,08,09,0 A, 0 B \ldots$. OZ, followed by 10-1Z, 20-2Z, etc.

The CID Number appears on each page of the manuscript. The complete citation is used on the first page, and an abbreviated version on subsequent pages. 


\title{
Contents
}

\author{
vii Authors \\ xi Conference Committee
}

\section{SESSION 1 CRYSTAL FIBER LASERS I}

934202 Single crystal fiber for laser sources (Invited Paper) [9342-1]

934203 High-power Yb:YAG single-crystal fiber amplifiers for femtosecond lasers [9342-2]

934204 Erbium distribution in single crystal YAG fibers grown by laser-heated pedestal growth technique [9342-3]

934205 Investigation of the amplification properties of Ho:YAG single crystal fiber [9342-4]

\section{SESSION 2 CRYSTAL FIBER LASERS II}

934207 Beam quality investigation in Nd:YAG crystal fiber amplifier pumped at >110w [9342-6]

934209 Laser diode pumped high efficiency Yb:YAG crystalline fiber waveguide lasers [9342-8]

$93420 \mathrm{~A}$ Templated growth of II-VI semiconductor optical fiber devices and steps towards infrared fiber lasers (Invited Paper) [9342-9]

\section{SESSION 3 EYE-SAFE AND MID-IR LASERS}

$9342 \mathrm{OB} \quad 4.5 \mathrm{~W}$ mid-infrared supercontinuum generation in a ZBLAN fiber pumped by a Q-switched mode-locked $\mathrm{Tm}^{3+}$ - doped fiber laser [9342-10]

$93420 \mathrm{C}$ Multi-wavelength resonant pumping of Er:YAG lasers for energy efficient trace gas detection systems [9342-11]

9342 OD Ho:YLF non-planar ring laser with fractional image rotation [9342-12]

9342 OE Gain-switched operation of ultrafast laser inscribed waveguides in Cr:ZnSe [9342-13]

9342 OF A continuous wave Fe:ZnSe laser pumped by efficient $\mathrm{Er}: \mathrm{Y}_{2} \mathrm{O}_{3}$ laser [9342-14]

9342 OG Radiation-enhanced thermal diffusion of transition metal and rare earth ions into II-VI semiconductors [9342-15] 
$9342 \mathrm{OH} \quad$ Radiation tests on erbium-doped garnet crystals for spaceborne CH4-Lidar applications [9342-16]

9342 Ol Multi-pulse detection technique to improve the timing/range resolution in a scanning LADAR system [9342-17]

$93420 \mathrm{~J} \quad$ Single frequency and wavelength stabilized near infrared laser transmitter for water vapor DIAL remote sensing application [9342-18]

9342 OK Monolithic solid-state lasers for spaceflight [9342-19]

9342 OL ICESat-2 laser technology readiness level evolution [9342-20]

9342 OM Laser amplifier development for IPDA Lidar measurements of $\mathrm{CO}_{2}$ from space [9342-21]

SESSION $5 \quad$ UV AND VISIBLE LASERS

9342 ON IW frequency-doubled VCSEL-pumped blue laser with high pulse energy [9342-22]

9342 OP Development of high coherence, $200 \mathrm{~mW}, 193 \mathrm{~nm}$ solid-state laser at $6 \mathrm{kHz}$ [9342-24]

$93420 Q \quad$ Annealing temperature dependence of random lasing properties in a diamond nanoparticle film [9342-25]

9342 OR Demonstration of miniaturized 20mW CW $280 \mathrm{~nm}$ and $266 \mathrm{~nm}$ solid-state UV laser sources [9342-26]

\section{SESSION 6 DISK LASERS}

9342 OT Front end for high-repetition rate thin disk-pumped OPCPA beamline at ELI-beamlines [9342-28]

9342 OU Thin-disk laser multi-pass amplifier [9342-29]

$9342 \mathrm{OV} \quad$ Ultrafast thin-disk multipass amplifier with $1.4 \mathrm{~kW}$ average power and $4.7 \mathrm{~mJ}$ pulse energy at $1030 \mathrm{~nm}$ converted to $820 \mathrm{~W}$ and $2.7 \mathrm{~mJ}$ at $515 \mathrm{~nm}$ [9342-30]

9342 OW First demonstration of passively mode-locked Yb:CaF 2 thin-disk laser [9342-31]

9342 OY Latest advances in high brightness disk lasers [9342-33]

SESSION 7 NOVEL CONCEPTS I

$93420 Z$ Double-beam, mode-controlling diode side-pumped Nd:YLF laser with near $60 \%$ efficiency [9342-34]

iv 
934210 LED side-pumped $\mathrm{Nd}^{3+}: \mathrm{YVO}_{4}$ laser at room temperature [9342-35]

\section{SESSION $8 \quad$ NOVEL CONCEPTS II}

934214 Fully vectorial laser resonator modeling by vector extrapolation methods [9342-39]

934216 High power tube solid-state laser with zigzag propagation of pump and laser beam [9342-41]

934217 In-phase synchronization of array laser using intra-Talbot-cavity second harmonic generation [9342-42]

SESSION 9 LASER MATERIALS AND CHARACTERIZATION

9342 1D Energy transfer upconversion measurements for popular neodymium-doped crystals [9342-49]

SESSION 10 ULTRAFAST LASERS

$9342 \mathrm{lE} \quad$ First experimental results towards a $100 \mathrm{~W}$ wavelength tunable femtosecond OPCPA [9342-50]

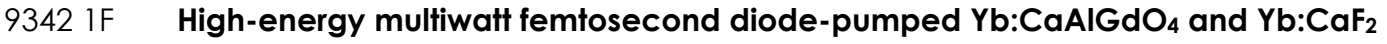
regenerative amplifiers [9342-51]

9342 1G Single grating mirror intracavity stretcher design for chirped pulse regenerative amplification [9342-52]

$9342 \mathrm{lJ} \quad$ High average power picosecond laser for selective material processing at $1342 \mathrm{~nm}$ wavelength [9342-55]

SESSION 11 PULSED LASERS I

$93421 \mathrm{~K} \quad 1 \mathrm{~mJ}$ single-rod fiber Er:glass laser for rangefinding [9342-56]

$93421 \mathrm{~L}$ A cryo-cooled high-energy DPSSL system delivering ns-pulses at $10 \mathrm{~J}$ and $10 \mathrm{~Hz}$ [9342-57]

SESSION 12 PULSED LASERS II

9342 1M Mode-locking in intracavity frequency doubled Nd:YVO4 laser [9342-59]

$93421 \mathrm{~N} \quad$ Current status of Kumgang laser system [9342-60]

$934210 \quad 50 W$ CW output power and $12 \mathrm{~mJ}$ pulses from a quasi-2-level Yb:YAG ceramic rod laser end-pumped at the $969 \mathrm{~nm}$ zero-phonon line [9342-61] 
9342 IP Investigation of mechanically Q-switched lasers [9342-62]

POSTER SESSION

$93421 Q \quad$ Numerical simulations of the optical gain of crystalline fiber doped by rare earth and transition ion [9342-63]

9342 is Temperature influence on diode pumped Er:CaF 2 laser [9342-67]

9342 IT Wavelength tunability of laser based on Yb-doped YGAG ceramics [9342-68]

$93421 \mathrm{U}$ On the efficiency of Tm-doped $2 \mu \mathrm{m}$ lasers [9342-69]

$93421 \mathrm{~V}$ Fe:ZnSe and Fe:ZnMgSe lasers pumped by Er:YSGG radiation [9342-70]

9342 IW Moderate high power 1 to $20 \mu$ s and kHz Ho:YAG thin disk laser pulses for laser lithotripsy [9342-71]

9342 1X Influence of temperature on spectroscopic and lasing properties of Pr:YLF crystal [9342-72]

9342 1Y 60W Ho:YLF oscillator-amplifier system [9342-73]

934212 Development of a closed-loop cryogenically cooled sub-picosecond regenerative amplifier [9342-74]

934220 Continuous-wave hybrid index-antiguided and thermal-guided planar waveguide laser with large mode area [9342-75]

$934221 \quad$ Underwater laser detection system [9342-76]

934222 Transmitted beam profile for determining bulk scattering in transparent ceramics [9342-77]

934223 Angle resolved scatter measurement of bulk scattering in transparent ceramics [9342-78]

934225 Spectroscopic characterization of $\mathrm{Cr}^{2+}$ ions in $\mathrm{ZnSe} / \mathrm{ZnS}$ crystals under visible excitation [9342-81]

934226 Direct measurement of up-conversion processes in diode pumped erbium-doped YAG [9342-82] 


\section{Authors}

Numbers in the index correspond to the last two digits of the six-digit citation identifier (CID) article numbering system used in Proceedings of SPIE. The first four digits reflect the volume number. Base 36 numbering is employed for the last two digits and indicates the order of articles within the volume. Numbers start with 00, 01, 02, 03, 04, 05, 06, 07, 08, 09, OA, OB...0Z, followed by 10-12, 20-2Z, etc.

Abdou Ahmed, Marwan, 03, OU, OV, OW, $1 \mathrm{G}$ Abshire, James B., OM

Adouane, A., 1Q

Agnesi, A., IF, 1G

Ahn, HeeKyung, iN

Aleknavicius, Aidas, 07

Alekseeva, Irina P., 1K

Alexeev, Sergey G., $1 \mathrm{M}$

Antipenkov, Roman, OT

Antognini, A., OU

Aravazhi, S., $1 U$

Asoubar, Daniel, 14

Aubourg, Adrien, 02

Aus-der-Au, J., IF, IG

Badding, John V., OA

Bakule, Pavel, OT

Balembois, François, 02, 03, 10

Banerjee, Saumyabrata, $1 \mathrm{~L}$

Barbet, Adrien, 10

Baril, Neil F., OA

Bartschke, Jürgen, 10

Batysta, František, OT

Baver, Dominik, OV

Beaurepaire, Julien, OR

Beecher, Stephen J., OE, ID

Bera, Subhabrata, 04

Berrou, A., OB

Berry, Patrick A., OE, OF

Betin, Alexander, $0 M$

Blanchot, Jean-Philippe, 10

Boubir, B., 1Q

Buchenkov, Vyacheslav A., 1K

Buckley, Ian, 05

Burnham, Ralph, 0J

Burns, Patrick M., OL

Butcher, Thomas J., $1 \mathrm{~L}$

Cadier, B., OB

Caracciolo, E., IF, IG

Casperson, Lee, 20

Chaudhuri, Subhasis, OA

Chazevskis, Gediminas, $1 \mathrm{~J}$

Chen, Tong, ON

Chuang, Ti, OJ

Cole, Brian, IP

Collier, John L., $1 \mathrm{~L}$

Cubera, Miroslaw, $\mathrm{OH}$

Dannecker, Benjamin, OW

Daovi, A. K., $1 Q$

De Vido, Mariastefania, 1L
Deana, Alessandro M., OZ

Délen, Xavier, 02, OW

Demagh, N., $1 Q$

Dementjev, Aleksandr S., 07

Deyra, Loïc, 02

Didierjean, Julien, 02, 03

Donelan, B., OB

Doroshenko, Maxim E., 1S, IV

Dreyer, Elizabeth F. C., 04

Druon, Frédéric, 10

Dymshits, Olga S., $1 \mathrm{~K}$

Edwards, Ryan E., OL

Eichhorn, Marc, OB, OD

Eichler, Hans J., OC

El-Sharkawy, Yasser H., 21

El-Sherif, Ashraf F., 21

Endo, Akira, 1T, $1 \mathrm{Z}$

Ertel, Klaus, $1 \mathrm{~L}$

Esser, Dominik, $\mathrm{OH}$

Evans, Jonathan W., OF

Fedorov, Pavel P., is

Fedorov, Vladimir V., OG, 25

Fibrich, Martin, $1 \mathrm{X}$

Fitzgibbons, Thomas C., OA

Fries, Christian, 10

Fritsche, Haro, OC

Fujiwara, Hideki, $O Q$

Gaertner, Martin, OC

Gafarov, Ozarfar, OG

García-Blanco, S. M., $1 U$

Georges, Patrick, 02, 03, 10

Georges, Thierry, OR

Gerasimenko, A. S., IV

Ghosh, Chuni, ON

Ghoumazi, M., 1Q

Glebov, Leonid, OK

Glebova, Larissa, OK

Goldberg, Lew, 1P

Gomaa, Walid, 21

Gomes, Jean Thomas, 03

Goorsky, Mark S., 22, 23

Gopalan, Venkatraman, OA

Gottwald, Tina, OY

Graf, Thomas, 03, 0U, 0V, 0W, IG

Grardel, Hugo, 10

Green, Jonathan T., OT

Greenhalgh, R. Justin S., 1 L

Gries, Wolfgang, OC

Grishin, Mikhail, $1 \mathrm{~J}$ 
Grivas, C., $1 \mathrm{U}$

Grohe, Andreas, OC

Gronloh, Bastian, $\mathrm{OH}$

Guandalini, A., 1F, IG

Hage, A., IE

Hänsch, T. W., OU

Harrington, James A., 04, 05

$\mathrm{He}$, Rongrui, OA

Healy, Noel, OA

Her, Tsing-Hua, 20

Hernandez-Gomez, Cristina, $1 \mathrm{~L}$

Hirosawa, Kenichi, 17

Hoef, Christopher D., 04

Höfer, Marco, $\mathrm{OH}$

Hoffmann, Hans-Dieter, $\mathrm{OH}$

Hönninger, Clemens, OW

Höppner, $\mathrm{H}_{\text {., }} 1 \mathrm{E}$

Horáček, Martin, OT

Hough, Nathaniel, IP

Hovis, Floyd E., OL

Ito, S., OP

Jacobs, Cobus, $1 Y$

Jambunathan, Venkatesan, IT, $1 \mathrm{Z}$

Jelínek, M., IV

Jelínková, Helena, 1S, 1T, 1V, 1X, 1Z

Johnson, Eric G., 05

Joulain, $F$., OB

Juhre, Ronny, OC

Kakizaki, K., OP

Kannari, Fumihiko, 17

Kar, Ajoy K., OE

Kemnitzer, M., 1F, 1G

Kharitonov, Artem A., IK

Kieleck, C., OB

Kienle, F., $1 G$

Killi, Alexander, OV, OY

Kim, Jom Sool, $1 \mathrm{~N}$

Kirch, K., OU

Kneis, C., OB

Koen, Wayne, IY

Komar, V. K., IV

Kong, Hong Jin, $1 \mathrm{~N}$

Kottmann, K., OU

Kovalenko, N. O., IV

Kovalev, Anton V., 1M

Krainak, Michael A., OK

Kreitler, Martin, $\mathrm{OH}$

Krishnamurthi, Mahesh, OA

Kubeček, V., IV

Kucirek, Philipp, $\mathrm{OH}$

Kuhn, Michael, 14

Kuhn, Vincent, OY

Landru, Nicolas, OR

Le Bail, Guy, OR

Le Guen, Bruno, OR

Lee, Hwihyeong, IN

Lesparre, Fabien, 02, 03

L'huillier, Johannes A., 10

Li, Yuan, 05

Linnemann, J., 1 Z
Litvinovitch, Viatcheslav, OL

Liu, Brad Chun-Ting, 26

Liu, Yuanye, 20

Loescher, André, 03, 0V

Losee, Andrew, 0J

Lucianetti, Antonio, $1 T$

Lux, Oliver, OC

Macdonald, John R., OE

Mackenzie, Jacob I., ID

Mak, Andrey A., 1K, IM

Manek-Hönninger, I., OB

Markushin, Yury Y., OI

Martial, Igor, 02, 03

Martinez, Alán, OG

Martyshkin, Dmitry, OG

Mason, Paul D., 1L

Mazanec, Tomáš, OT

McDaniel, Sean A., OE

Meissner, Ansgar, $\mathrm{OH}$

Meissner, Helmuth, 09, OK

Meissner, Stephanie, 09, OK

Melzer, Jeffrey E., 04

Merritt, Scott, OK

Michailovas, Andrejus, 07, $1 \mathrm{~J}$

Miller, Jerome Keith, 05, 22, 23

Mirov, Sergey B., OG, 25

Miura, Taisuke, 1T, $1 \mathrm{Z}$

Mocek, Tomáš, 1T, $1 Z$

Mu, Xiaodong, 09, OK

Nakazato, T., OP

Naylon, Jack A., OT

Negel, Jan-Phillipp, 03, 0V

Němec, Michal, 1S, 1V

Nettleton, John, IP

Nie, Craig D., 04, 05

Niyuki, Ryo, OQ

Novák, Jakub, OT

Oh, Jungsuk, iN

Onose, T., OP

Orlov, Oleg A., $1 \mathrm{M}$

Osiko, Vyacheslav V., is

Pallmann, Wolfgang, 03

Park, Sangwoo, $1 \mathrm{~N}$

Pati, Gour S., Ol

Paul, Amandine, 10

Peacock, Anna C., OA

Peppers, Jeremy M., 25

Phillips, P. Jonathan, 1L

Pirzio, F., IF, IG

Pohl, R., OU

Pollnau, M., $1 \mathrm{U}$

Polyakov, Vadim M., IK, $1 \mathrm{M}$

Poulain, M., OB

Prandolini, M. J., $1 \mathrm{E}$

Puffenberger, Kent, 0J

Puzikov, V. M., IV

Rand, Stephen C., 04

Renz, Günther, 1 W

Resan, Bojan, 03

Richards, David, $1 \mathrm{~L}$ 
Riedel, R., $1 \mathrm{E}$

Robin, T., OB

Rodin, Aleksej M., 07, $1 \mathrm{~J}$

Rodionov, Andrey YU., $1 \mathrm{~K}$

Rumpel, M., $1 G$

Rus, Bedřich, OT

Ryasnyanskiy, Aleksandr, OK

Ryba, Tracey, OY

Sanamyan, Tigran, OF

Sarukura, N., OP

Sasaki, Keiji, OQ

Savich, Michael, 16

Sawruk, Nicholas W., OL

Sazio, Pier J. A., OA

Schad, Sven-Silvius, OY

Schellhorn, Martin, OD

Schepler, Kenneth L., OE

Schuett, Casey, OC

Schuhmann, K., OU

Schulz, M., 1E

Schum, Tom, OJ

Seurin, Jean-Francois, ON

Shaikh, Waseem, $1 \mathrm{~L}$

Sharma, Saurabh, 22, 23

Shohda, Fumio, 17

Shori, Ramesh K., 05, 22, 23, 26

Shuman, Tim, OJ

Sikocinski, P., 12

Smirnov, Vadim, OK

Smith, Jodie M., $1 \mathrm{~L}$

Sparks, Justin R., OA

Stafsudd, Oscar M., 26

Stephen, Mark A., OK

Stolzenburg, Christian, OY

Storm, Mark, OM

Strauss, Hencharl J., IY

Šulc, Jan, 1S, 1T, 1V, 1X

Sullivan, Edward, OL

Sutter, Dirk H., OV

Švejkar, Richard, 1S

Takashima, Hideaki, $\mathrm{OQ}$

Tanaka, Y., OP

Taqqu, D., OU

Tavella, F., 1E

Theobald, Christian, 10

Trembath-Reichert, Stephen, 04

Tripathi, Renu, 0

Tsuboi, M., OP

Ulevichius, Nortautas, $1 \mathrm{~J}$

v. Löwis of Menar, Patric, 10

van Dalfsen, K., $1 \mathrm{U}$

Van Leeuwen, Robert, ON

VanTuijl, Andre, OL

Vitkin, Vladimir V., 1K, $1 \mathrm{M}$

Voss, Andreas, OU, OV, OW

Walters, Brooke, 0J

Wang, Qing, ON

Watanabe, S., OP

Watkins, Laurence, ON

Weichelt, Birgit, OU, OW
Weitz, Marco, 10

Wentsch, Katrin S., OW

Wetter, Niklaus U., OZ

Williams, Lamario, OG

Wu, Lorinda, IY

Wyrowski, Frank, 14

Wysocki, Theodore, $\mathrm{OL}$

XU, Guoyang, ON

Yan, RenPeng, 1D

Yanagisawa, Takayuki, 17

Yoon, Sung Jin, ID

Yu, Anthony W., OK, OM

Zagorviko, Y. A., IV

Zervos, Charalampos, OT

Zhang, Zeyu, 05

Zhilin, Alexander A., 1K

Zhou, Delai, ON 
Proc. of SPIE Vol. $9342934201-10$

Downloaded From: https://www.spiedigitallibrary.org/conference-proceedings-of-spie on 26 Apr 2023 Terms of Use: https://www.spiedigitallibrary.org/terms-of-use 


\title{
Conference Committee
}

\author{
Symposium Chairs
}

Guido Hennig, Daetwyler Graphics AG (Swtizerland)

Yongfeng Lu, University of Nebraska-Lincoln (United States)

Symposium Co-chairs

Bo Gu, Bos Photonics (United States)

Andreas Tünnermann, Fraunhofer-Institut für Angewandte Optik und Feinmechanik (Germany) and Friedrich-Schiller-Universität Jena (Germany)

Program Track Chair

Gregory J. Quarles, Optoelectronics Management Network (United States)

\section{Conference Chairs}

W. Andrew Clarkson, University of Southampton (United Kingdom) Ramesh K. Shori, SPAWAR Systems Center (United States)

\section{Conference Program Committee}

Patrick A. Berry, Air Force Research Laboratory (United States)

Marc Eichhorn, Institut Franco-Allemand de Recherches de SaintLouis (France)

Dennis G. Harris, MIT Lincoln Laboratory (United States)

Norman Hodgson, Coherent, Inc. (United States)

Helena Jelínková, Czech Technical University in Prague (Czech Republic)

Christian Kränkel, Universität Hamburg (Germany)

Jacob I. Mackenzie, University of Southampton (United Kingdom)

Markus Pollnau, Universiteit Twente (Netherlands)

Narasimha S. Prasad, NASA Langley Research Center (United States)

David H. Titterton, Defence Science and Technology Laboratory (United Kingdom)

Masaki Tokurakawa, The University of Electro-Communications (Japan)

Matteo Vannini, Istituto Nazionale di Ottica, CNR (Italy) 


\section{Session Chairs}

1 Crystal Fiber Lasers I

Ramesh K. Shori, SPAWAR Systems Center (United States)

2 Crystal Fiber Lasers II

Ramesh K. Shori, SPAWAR Systems Center (United States)

3 Eye-Safe and Mid-IR Lasers

Patrick A. Berry, Air Force Research Laboratory (United States)

$4 \quad$ Airborne and Space Qualified Lasers

Narasimha S. Prasad, NASA Langley Research Center (United States)

5 UV and Visible Lasers

Dennis G. Harris, MIT Lincoln Laboratory (United States)

6 Disk Lasers

Jacob I. Mackenzie, University of Southampton (United Kingdom)

7 Novel Concepts I

Ramesh K. Shori, SPAWAR Systems Center (United States)

8 Novel Concepts II

Jacob I. Mackenzie, University of Southampton (United Kingdom)

9 Laser Matericals and Characterization

Ramesh K. Shori, SPAWAR Systems Center (United States)

10 Ultrafast Lasers

Jacob I. Mackenzie, University of Southampton (United Kingdom)

11 Pulsed Lasers I

W. Andrew Clarkson, University of Southampton (United Kingdom)

12 Pulsed Lasers II

W. Andrew Clarkson, University of Southampton (United Kingdom) 